\title{
Assessment of afoxolaner efficacy against Otodectes cynotis infestations of dogs
}

\author{
Doug Carithers ${ }^{1 *}$, Jordan Crawford ${ }^{1}$, Christa de Vos ${ }^{2}$, Alta Lotriet $^{2}$ and Josephus Fourie ${ }^{2}$
}

\begin{abstract}
Background: The efficacy of a single $2.5 \mathrm{mg} / \mathrm{kg}$ dose of afoxolaner (NexGard ${ }^{\oplus}$, Merial) against induced Otodectes cynotis infestations was assessed in eight afoxolaner-treated dogs, compared to eight untreated dogs.

Methods: After O. cynotis infestations were established and confirmed by otoscopic assessments in 16 dogs, all of the dogs were included in the study and allocated to two separate treatment groups. The first group of eight ear mite-infested dogs remained untreated, while afoxolaner was administered orally to the second group of dogs at the minimum recommended dose once on Day 0. Otoscopic assessments performed on all dogs (Days $-7,-2,14$ and 28) confirmed the presence or absence of live mites throughout the study. No serious adverse events were recorded throughout the study, and no adverse events were likely related to the administration of NexGard.
\end{abstract}

Results: By Day 28, seven out of eight untreated dogs were still infested with ear mites, while only two out of eight afoxolaner-treated dogs were infested, with one and four ear mites, respectively. On Day 28, the reductions of mite counts in the afoxolaner-treated group versus those of the control dogs were $98.5 \%$ based on geometric means, and $99.4 \%$ based on arithmetic means. Significantly fewer $(P<0.05)$ live mites were present in the afoxolaner-treated group than the untreated group on Day 28.

Conclusion: The results of this study demonstrated that a single oral administration of afoxolaner at the minimum recommended dose is highly effective (>98\%) in treating dogs with induced O. cynotis infestations.

Keywords: Otodectes cynotis, Dog, Afoxolaner, NexGard

\section{Background}

The ear mite Otodectes cynotis, the causative organism of otodectic mange [1], is a very common nonburrowing, surface-living [2] acarian which infests cats, dogs and many other carnivores. Otodectes cynotis is found all over the world and primarily infests the ear canals of its host; however, it has also been found on head, back and tail in heavy infestations [1,2]. The irritation caused by the mites often results in the host scratching its ears and shaking its head, which can lead to lacerations associated with the affected ear(s) or the formation of an auricular hematoma. Secondary yeast and bacterial infections often occur in the presence of heavy ear mite infestations.

Transmission of ear mites occurs by direct contact with an infested host [1-3], most commonly with the

\footnotetext{
* Correspondence: doug.carithers@Merial.com

'Merial, Inc, 3239 Satellite Blvd, Duluth 30096, GA, USA

Full list of author information is available at the end of the article
}

transmission of the mites from mother to kittens or puppies before weaning. Clinical signs which can lead to the suspicion of otodectic mange include bilaterally pruritic otitis with dry, blackish-brown cerumen in the host's ear canal; self-induced ear lesions; aural hematomas; and pruritic lesions on the face and neck. Diagnosis is usually made through otoscopic examination of the ear and microscopic examination of cerumen, which usually contains ear mites of all life stages (eggs, larvae, nymphs and adults). Additionally, skin scrapings can occasionally reveal ear mites [3]. It is important to note that, if at least one pet in the household is infested with ear mites, all pets in the household need to be treated for ear mites, as the mites are highly contagious $[1,4]$.

Since O. cynotis is a very common parasite of dogs and cats, it is essential to find effective treatments for infested pets. Historically, treatment necessitated ear flushing and cleansing with a mild ceruminolytic agent. Typical treatments include mineral oil or acaricides 
(monosulfiram, permethrin, or amitraz [3, 4]) directly administered into the ear canal.

Sometimes treating the ears alone would not solve the issue. Often to achieve control one would also treat the whole body with an acaricide dip or topical treatment, as mites can infest other parts of the body and re-infest the ears [3]. Later, systemic-topical treatments such as selamectin and imidacloprid/moxidectin spot on formulations were found to have good systemic activity against ear mites in the ear canal and on the body $[2,3,5,6]$.

Recently, a new class of insecticides/acaricides, the isoxazolines, has demonstrated excellent efficacy against $O$. cynotis and other similar parasites [7]. Afoxolaner is an isoxazoline administered monthly to protect dogs against fleas and ticks $\left(\mathrm{NexGard}{ }^{\circ}\right)$ [7-9]. It is administered at a minimum dose of $2.5 \mathrm{mg} / \mathrm{kg}$, and comparative studies have shown that monthly administrations of afoxolaner provide outstanding protection against fleas and ticks $[10,11]$. Afoxolaner has also demonstrated to be highly efficacious against Sarcoptes scabiei [12] and Demodex canis [13, 14]. Based on these observations, the present study was undertaken to assess the efficacy of a single oral administration of afoxolaner (NexGard) against $O$. cynotis infestations in dogs.

\section{Methods}

\section{Animals}

All dogs were a part of a colony at the study site, Clinvet, and were returned to the colony holding facility at the conclusion of the study. All animals were managed similarly and with due regard for their well-being. Trained personnel under the supervision of a veterinarian were responsible for the health of the dogs. All personnel involved in health assessments, data collection and physical examinations were blinded to the treatment allocation of the dogs. To prevent potential bias or unmasking, the treatment assessments were conducted in a non-systematic order. All dogs considered were over 6 months of age (age on day of treatment ranged from 2 years and ten months to 8 years and six months), and acclimatized to the study facility for at least seven days prior to being artificially infested with $O$. cynotis mites. Experimental infection was performed by harvesting mites by lavage from donor animals with patent natural infestations, and transferring approximately 50 to 100 mites, depending on intensity of infection in donor animals, into each ear of the recipient animal. From this pool of $O$. cynotis infested dogs, 16 dogs that ultimately developed established infections and also met qualification parameters as verified by a veterinarian via clinical examinations (Days -7 and -2) were included in the study. The study dogs weighed between 15.4 and $22.0 \mathrm{~kg}$ (as weighed on Day -1) (Table 1). The dogs were of mixed breed (all mongrels), and were healthy at the initiation of the study,
Table 1 Individual dog information/details, as assessed on Day -1

\begin{tabular}{|c|c|c|c|c|c|}
\hline \multicolumn{3}{|c|}{ Group 1 (negative control) } & \multicolumn{3}{|c|}{ Group 2 (afoxolaner-treated) } \\
\hline Animal ID & Sex & Body weight $(\mathrm{kg})$ & Animal ID & Sex & Body weight $(\mathrm{kg})$ \\
\hline 4D9 16A & M & 17.8 & 287466 & $\mathrm{~F}$ & 15.4 \\
\hline $4 \mathrm{DE} 28 \mathrm{~F}$ & $\mathrm{~F}$ & 18.4 & CC3 CEE & M & 19.8 \\
\hline $86 C 5 B 7$ & M & 22.0 & CD5 B63 & $\mathrm{F}$ & 18.4 \\
\hline $95636 \mathrm{~F}$ & $\mathrm{~F}$ & 20.2 & DF4 840 & M & 17.2 \\
\hline B2C 7B5 & $\mathrm{F}$ & 18.6 & DF5 29E & M & 17.0 \\
\hline CC5 7C6 & $\mathrm{F}$ & 16.6 & E15 130 & M & 20.8 \\
\hline DF5 E10 & M & 20.2 & E17 62E & M & 18.2 \\
\hline DF7 CD8 & M & 21.4 & E19 161 & $\mathrm{~F}$ & 19.8 \\
\hline \multicolumn{3}{|c|}{$\begin{array}{l}\text { Arithmetic mean body } \\
\text { weight in kg: } 19.4\end{array}$} & \multicolumn{3}{|c|}{$\begin{array}{l}\text { Arithmetic mean body } \\
\text { weight in kg: } 18.3\end{array}$} \\
\hline
\end{tabular}

except for clinical signs associated with the induced $O$. cynotis infestations. Nine males and seven females were included in the study, and the females were not pregnant or lactating. Once enrolled, dogs were kept individually in cages under strict isolation conditions, with no physical contact between animals possible. None of the dogs included in the study had been treated with a long-lasting topical or systemic miticidal/acaricidal/insecticidal product during the 12 weeks preceding Day 0, nor had they been treated with any isoxazolines for 6 months prior to Day 0.

\section{Study design and treatment}

This study was a randomized, single center, controlled efficacy study, following a randomized block design, and all evaluations of efficacy were performed by personnel in blinded conditions. Each dog was an experimental unit. For each dog, a total of $>10$ live mites in at least one ear, as well as the presence of $\geq 1$ live mite in the other ear, was necessary for inclusion in the study. Dogs were enrolled as they qualified, thus Day 0 was not the same calendar day for all dogs.

Once qualified, dogs were ranked within sex, in descending order of individual pre-administration (Day -2) live mite count, and were subsequently blocked into eight replicates of two dogs each. The dogs were then allocated to untreated group 1 and treated group 2. Dogs were weighed on Day -1 to ensure that, as close as possible and without under-dosing, the labeled minimum dose of afoxolaner $(2.5 \mathrm{mg} / \mathrm{kg})$ was administered using combinations of intact commercially available product (Table 2). All dogs were observed hourly for four hours post-administration, for possible adverse events from product administration.

Otoscopic assessments were performed on all dogs on Days $-7,-2,14$ and 28 to confirm the initial presence, and subsequent presence or absence, of live mites. 
Table 2 Administration of NexGard (afoxolaner) at $~ 2.5 \mathrm{mg} / \mathrm{kg}$ to the afoxolaner-treated group (Group 2) on Day 0

\begin{tabular}{lclc}
\hline Animal ID & $\begin{array}{l}\text { Body weight } \\
(\mathrm{kg})\end{array}$ & Chews administered & $\begin{array}{c}\text { Actual final } \\
\text { dose }(\mathrm{mg} / \mathrm{kg})\end{array}$ \\
\hline 2003287 466 & 15.4 & $1 \times 28.3 \mathrm{mg} \& 1 \times 11.3 \mathrm{mg}$ & 2.57 \\
CC3 CEE & 19.8 & $1 \times 28.3 \mathrm{mg} \& 2 \times 11.3 \mathrm{mg}$ & 2.57 \\
CD5 B63 & 18.4 & $1 \times 28.3 \mathrm{mg} \& 2 \times 11.3 \mathrm{mg}$ & 2.77 \\
DF4 840 & 17.2 & $4 \times 11.3 \mathrm{mg}$ & 2.63 \\
DF5 29E & 17.0 & $4 \times 11.3 \mathrm{mg}$ & 2.66 \\
E15 130 & 20.8 & $5 \times 11.3 \mathrm{mg}$ & 2.72 \\
E17 62E & 18.2 & $1 \times 28.3 \mathrm{mg} \& 2 \times 11.3 \mathrm{mg}$ & 2.80 \\
E19 161 & 19.8 & $1 \times 28.3 \mathrm{mg} \& 2 \times 11.3 \mathrm{mg}$ & 2.57 \\
\hline
\end{tabular}

\section{Ear flushing and mite counts}

On Day 28, a qualitative assessment of the ear canals was performed prior to the flushing procedure to determine the presence of viable mites. This was followed by the quantitative assessment of ear mites by ear duct flushing, complete mite collection and count. The ear ducts were filled with an appropriate solution of a $5 \%$ aqueous solution of docusate sodium ( 3 to $4 \mathrm{ml}$ per ear canal), then the ears massaged externally until sufficient melting of the ear duct content was observed. The melted solution for each ear was collected into a labeled collection container, followed by a saline flush of the ear duct into the same container. The contents of the container were drained into a $38 \mu \mathrm{m}$ sieve, which was then rinsed with clean water immediately, and the contents for each ear were transferred separately into labeled bottles. This procedure was conducted on each ear until otoscopic examinations revealed that both ears were clean. The contents of each ear were collected, examined, and stored separately in the labeled bottles. All collected material was observed microscopically on the same day, and all observed live mites (adult and immature) were counted and recorded for each ear.

\section{Statistical analysis}

The primary efficacy criterion was the number of live mites collected from the NexGard-treated group on Day 28 , compared to that of the untreated control group on the same day. The statistical unit was the individual animal. The average percent reduction in mite counts for each group was calculated using geometric means: Efficacy $(\%)$ against ear mites $=100 \times($ GMC - GMT $) / G M C$, where GMC is the geometric mean number of live mites in dogs in the untreated group and GMT is the geometric mean number of live mites in dogs in the treated group. The groups were compared using a one-way ANOVA (Proc GLM procedure in SAS) with an administration effect after a logarithmic transformation on the mite (count +1$)$ data, and by using an ANOVA (Proc
GLM procedure in SAS) with an administration effect on untransformed mite count data.

Additionally, due to large variability in mite counts in the negative control group, a non-parametric alternative test (the Mann-Whitney $U$-test) was used to assess efficacy by comparing median values. SAS version 9.3 TS Level 1 M2 [15] was used for all statistical analyses. Statistical significance for geometric mean and arithmetic mean assessments was declared at a two-sided $P$-value of 0.05 .

\section{Results}

\section{Clinical examinations and health observations}

None of the clinical signs (mild pruritus, intermittent head-shaking) associated with the mite infestations observed during any of the clinical examinations were deemed as serious or affecting overall health and wellbeing. The examining veterinarian carefully examined and judged all enrolled dogs to be fit to continue in the study, at each assessment throughout the study. No serious health issues were recorded during the study, with only mild signs consistent with $O$. cynotis infestation observed. No adverse events related to the administration of afoxolaner were observed.

\section{Antiparasitic efficacy}

All of the dogs were positive for the presence of ear mites prior to Day 0 of the study. For the untreated control dogs (Group 1), the number of live mites found on each dog on Day 28 ranged from 0 to 477 . For the afoxolaner-treated dogs (Group 2), one dog harbored one mite, another had four live mites, with the remaining six dogs having no live mites found by Day 28. Based on geometric means, an average of 22.3 live mites per negative control dog, and an average of 0.3 live mites per afoxolaner-treated dog, was recorded after ear flushing. Calculating based on arithmetic means, an average of 109.9 live mites per negative control dog, and an average of 0.6 live mites per afoxolaner-treated dog, was recorded after ear flushing. Based on both geometric (one-way ANOVA with a treatment effect) means (ANOVA: $F_{(1,14)}=11.74, P=0.0041$ ) and arithmetic (Mann-Whitney $U$-test) assessments $\left(W_{(1)}\right.$ =43.5, $Z=-2.6354, P=0.0084$ ) significantly fewer live mites were present in the afoxolaner-treated group than were present in the untreated group by Day 28. The percent efficacy of NexGard (afoxolaner) against induced Otodectes cynotis on Day 28 was calculated to be $98.5 \%$ based on geometric means and $99.4 \%$ based on arithmetic means (Table 3).

\section{Discussion}

Otodectes cynotis is a commonly occurring surfacedwelling mite that can easily transfer between suitable hosts [1-3]. Since these mites can exist on infected hosts 
Table 3 Efficacy against Otodectes cynotis based on geometric mean and arithmetic median number of mites recovered from both Group 1 (negative control) and Group 2 (afoxolanertreated) dogs

\begin{tabular}{|c|c|c|c|c|c|}
\hline \multicolumn{3}{|c|}{ Geometric } & \multicolumn{3}{|c|}{ Arithmetic } \\
\hline $\begin{array}{l}\text { Group } 1 \\
\text { mean }\end{array}$ & $\begin{array}{l}\text { Group } 2 \text { mean } \\
\text { (\% efficacy) }\end{array}$ & $P$-value & $\begin{array}{l}\text { Group } 1 \\
\text { mean }\end{array}$ & $\begin{array}{l}\text { Group } 2 \text { mean } \\
\text { (\% efficacy) }\end{array}$ & $P$-value \\
\hline 22.3 & $0.3(98.5)$ & $0.0041^{a}$ & 109.9 & $0.6(99.4)$ & $0.0084^{b}$ \\
\hline
\end{tabular}

ane-way ANOVA: $F_{(1,14)}=11.74, P=0.0041$

${ }^{b}$ Non-parametric analysis using the Mann-Whitney test: $W_{(1)}=43.5$,

$Z=-2.6354, P=0.0084$

outside the ear canals $[1,2]$, in order to effectively treat existing infestations, or potentially prevent infestations, effective systemic acaricidal products should be utilized. Properly ascertaining the overall efficacy of antiparasitic products often requires multiple studies be performed. Typically, the first such study performed is a controlled laboratory study utilizing the minimum target dose, realizing that studies of different scales will follow, should the results indicate a potential for success.

This study was designed to assess the efficacy of the minimum dose of afoxolaner against existing infestations of O. cynotis in dogs under controlled, laboratory conditions. The present study demonstrates that treatment with a single oral dose (at or approaching the minimum recommended dosage of $2.5 \mathrm{mg} / \mathrm{kg}$ ) of afoxolaner (NexGard ${ }^{\circ}$ Chewables) resulted in a rapid reduction in ear mite numbers, suggesting that a single oral administration of NexGard is highly effective (>98\%) against O. cynotis infestations in dogs, as assessed 28 days post-administration. This is comparable to efficacy achieved when afoxolaner was tested against other mite species infesting dogs, namely Sarcoptes scabiei (100\% efficacy based on mite counts) [12] and Demodex spp., with $>99 \%$ efficacy based on mite counts in a well-controlled study [12] and resolution of cases under field conditions [14].

\section{Conclusion}

The high level of efficacy achieved with a single, minimum dose of NexGard ${ }^{\circ}$ (afoxolaner) in the present study would seem to justify additional work to gather further evidence of the treatment and control capabilities of afoxolaner against O. cynotis, also when considering high efficacy obtained against other mite species infesting dogs.

\section{Acknowledgements}

The authors would like to thank L. van Zyl and H. Jooste for the statistical analysis.

\section{Funding}

This clinical study was funded by Merial, Inc.

\section{Authors' contributions}

$\mathrm{DC}, \mathrm{JF}, \mathrm{CdV}$, and AL designed the study protocol. JF, CdV and AL were responsible for the conduct of the study. DC and JC drafted the first version of the manuscript, which was substantially revised by all authors. All authors read and approved the final manuscript.

\section{Competing interests}

Doug Carithers is an employee of Merial, Inc., and Jordan Crawford is a contractor for Merial Inc. ClinVet, of which Josephus Fourie, Christa de Vos and Alta Lotriet are employees, is an independent South African Contract Research Organization contracted to conduct the study. All authors voluntarily publish this article and have no personal interest in these studies, other than publishing the scientific findings in which they have been involved via planning, initiating, monitoring, and conducting the investigations and analyzing the results.

\section{Consent for publication}

Not applicable.

\section{Ethics approval}

The design and conditions of this study were approved by the Merial Institutional Animal Care and Use Committee (IACUC) and the local Animal Welfare Ethics Committee in accordance with Good Clinical Practice by the European Agency for the Evaluation of Veterinary Medicinal Products (CVMP/ VICH GL9, July 2000; CVMP/NICH GL19, July 2001).

\section{Disclaimer}

This document is provided for scientific purposes only. Any reference to a brand or a trademark herein is for informational purposes only and is not intended for a commercial purpose or to dilute the rights of the respective owner(s) of the brand(s) or trademark(s).

\section{Author details}

${ }^{1}$ Merial, Inc, 3239 Satellite Blvd, Duluth 30096, GA, USA. ${ }^{2}$ Clinvet International (Pty) Ltd, PO Box 111869321, Universitas, South Africa.

Received: 3 October 2016 Accepted: 1 December 2016

Published online: 09 December 2016

\section{References}

1. Wall R, Shearer D. Mites (Acari). In: Wall R, Shearer D, editors. Veterinary entomology: Arthropod ectoparasites of veterinary importance. Oxford: Springer; 2012. p. 70-2.

2. Shanks DJ, McTier TL, Rowan TG, Watson P, Thomas CA, Bowman DD, et al. The efficacy of selamectin in the treatment of naturally acquired aural infestations of Otodectes cynotis on dogs and cats. Vet Parasitol. 2000;91:283-90

3. Guaguère E. Ectoparasitic skin diseases. In: Guaguère E, Prelaud P, Craig M, editors. A practical guide to feline dermatology. Paris: Merial; 1999. p. 30.

4. Curtis CF. Current trends in the treatment of Sarcoptes, Cheyletiella and Otodectes mite infestations in dogs and cats. Vet Dermatol. 2004;15:108-14.

5. Arther RG. Mites and lice: Biology and control. In: Lindsay DS, Zajac AM, Editors. Veterinary clinics of North America. Philadelphia: W. B. Saunders; 2009. 39:6;1159-71.

6. Krieger K, Heine J, Dumont P, Hellmann K. Efficacy and safety of imidacloprid $10 \%$ plus moxidectin $2.5 \%$ spot-on in the treatment of sarcoptic mange and otoacariosis in dogs: results of a European field study. Parasitol Res. 2005;97:581-8.

7. Shoop WL, Hartline EJ, Gould BR, Waddell ME, McDowell RG, Kinney JB, et al. Discovery and mode of action of afoxolaner, a new isoxazoline parasiticide for dogs. Vet Parasitol. 2014;201:179-89.

8. European Medicines Agency. 2014. Nexgard summary of product characteristics. http://www.ema.europa.eu/docs/en_GB/document_library/ EPAR_-_Product_Information/veterinary/002729/WC500164067.pdf. Accessed 31 Aug 2015.

9. Halos L, Lebon W, Chalvet-Monfray K, Larsen D, Beugnet F. Immediate efficacy and persistent speed of kill of a novel oral formulation of afoxolaner $\left(\right.$ NexGard ${ }^{\oplus}$ ) against induced infestations with Ixodes ricinus ticks. Parasit Vectors. 2014;7:452.

10. Beugnet F, Liebenberg J, Halos L. Comparative efficacy of two oral treatments for dogs containing either afoxolaner or fluralaner against 
Rhipicephalus sanguineus sensu lato and Dermacentor reticulatus. Vet Parasitol. 2015;209:142-5.

11. Beugnet F, Liebenberg J, Halos L. Comparative speed of efficacy against Ctenocephalides felis of two oral treatments for dogs containing either afoxolaner or fluralaner. Vet Parasitol. 2015;207:297-301.

12. Beugnet F, de Vos C, Liebenberg J, Halos L, Larsen D, Fourie J. Efficacy of afoxolaner in a clinical field study in dogs naturally infested with Sarcoptes scabiei. Parasite. 2016;23:26.

13. Beugnet F, Halos L, Larsen D, de Vos C. Efficacy of oral afoxolaner for the treatment of canine generalised demodicosis. Parasite. 2016;23:14.

14. Chavez F. Case report of afoxolaner treatment for canine demodicosis in four dogs naturally infected with Demodex canis. J Appl Res Vet Med. 2016;14((2):123-7.

15. SAS Institute Inc. 2011. SAS 9.3 Statements: Reference. Cary, NC: SAS Institute Inc. SAS 9.3 Statements: Reference Copyright $\odot$ 2011, SAS Institute Inc., Cary, NC, USA ISBN 978-1-60764-902-1 (electronic book).

Submit your next manuscript to BioMed Central and we will help you at every step:

- We accept pre-submission inquiries

- Our selector tool helps you to find the most relevant journal

- We provide round the clock customer support

- Convenient online submission

- Thorough peer review

- Inclusion in PubMed and all major indexing services

- Maximum visibility for your research

Submit your manuscript at www.biomedcentral.com/submit
Biomed Central 